\title{
Managing acute ischaemic stroke in a small island developing state: meeting the guidelines in Barbados
}

\author{
Asanchia S. Harewood-Marshall', Leslie S. Craig ${ }^{1}$, Tanya P. Martelly ${ }^{1}$, David O. C. Corbin', Lauren Maul', \\ Damani McIntosh-Clarke', Tracey Blackman², Kenneth S. George ${ }^{2}$, Anselm J. M. Hennis' ${ }^{1}$ Ian R. Hambleton ${ }^{1}$ \\ and Angela M. C. Rose ${ }^{1 *}$
}

\begin{abstract}
Background: We describe hospital-based management of acute ischaemic stroke patients in 2010-2013 in Barbados, by comparing documented treatment given in the single tertiary public hospital with international guideline recommendations.
\end{abstract}

Methods: Evidence-based stroke management guidelines were identified through a systematic literature search. Comparisons were made between these guidelines and documented diagnostic practice (all strokes) and prescribed medication (ischaemic stroke only), using a combination of key informant interviews and national stroke registry data for 2010-2013.

Results: Several published international guidelines for the acute management of ischaemic stroke recommended patient management in a dedicated stroke unit or nearest hospital specialised in stroke care. Further, patients should receive clinical diagnosis, CT brain scan, specialist evaluation by a multidisciplinary team and, if eligible, thrombolysis with alteplase within 3-3.5 h of symptom onset. Subsequent secondary prophylaxis, with a platelet aggregation inhibitor and a statin was advised.

Barbados had no stroke unit or stroke team, and no official protocol for acute stroke management during the study period. Most of the 1735 stroke patients were managed by emergency physicians at presentation; if admitted, they were managed on general medical wards. Most had a CT scan (1646; 94.9\%). Of 1406 registered ischaemic stroke patients, only $6(0.4 \%)$ had been thrombolysed, 521 (37.1\%) received aspirin within $24 \mathrm{~h}$ of admission and 670 (47.7\%) were prescribed aspirin on discharge.

Conclusions: Acute ischaemic stroke diagnosis was consistent with international recommendations, although this was less evident for treatment. While acknowledging the difficulty in implementing international guidelines in a low-resource setting, there is scope for improvement in acute ischaemic stroke management and/or its documentation in Barbados. A stroke unit was established in August 2013 and written clinical protocols for acute stroke care were in development at the time of the study; future registry data will evaluate their impact. Our findings have implications for other low-resource settings with high stroke burden.

Keywords: Acute stroke, Guideline, Registry, Surveillance

\footnotetext{
* Correspondence: angela.rose@cavehill.uwi.edu

${ }^{1}$ George Alleyne Chronic Disease Research Centre, Caribbean Institute for

Health Research, The University of the West Indies, Cave Hill Campus,

Barbados

Full list of author information is available at the end of the article
}

(c) The Author(s). 2018 Open Access This article is distributed under the terms of the Creative Commons Attribution 4.0 International License (http://creativecommons.org/licenses/by/4.0/), which permits unrestricted use, distribution, and reproduction in any medium, provided you give appropriate credit to the original author(s) and the source, provide a link to the Creative Commons license, and indicate if changes were made. The Creative Commons Public Domain Dedication waiver (http://creativecommons.org/publicdomain/zero/1.0/) applies to the data made available in this article, unless otherwise stated. 


\section{Background}

Chronic non-communicable diseases (NCDs) are the leading cause of morbidity and mortality worldwide [1] and in many developing states, particularly in the Caribbean. A large proportion of this mortality is attributable to cardiovascular disease (CVD), including stroke [2-4].

In 2008, 82\% of deaths in Barbados (a small-island nation in the Caribbean with a population of about 278,000 in 2010 [5]) were due to NCDs [6]. Of these, $31 \%$ were attributable to CVD, with stroke second only to ischaemic heart disease [6]. Barbados, the fifteenth most densely populated nation in the world [7], is 21 miles long by 14 miles wide, with its single tertiary public hospital situated in the southwest of the island.

In 2008, The University of the West Indies, in collaboration with the Barbados Ministry of Health, implemented a population-based, multi-NCD registry, collecting data on all cases of stroke, heart attack and cancer island-wide [8]. Data for 2009-2013 from the stroke component of this Barbados National Registry for Chronic Non-communicable Disease (the BNR-Stroke) show an incidence rate age-standardised to the World Health Organization (WHO) world 2000 population for all strokes on the island (i.e. not just first-ever events) to be 153 per 100,000 (95\% CI 141-166), with age-standardised mortality of 79.3 (95\% CI 70.7-88.7) [9] for the same period.

For survivors of stroke, neurological deficits can persist, resulting in profound morbidity and dependence [1,9] as well as considerable health care costs [10-12] and loss of productivity, which impact at both individual and country levels $[10,11]$. Prompt and efficacious management of acute stroke is an important determinant of patient outcome [12] and the efficient allocation of medical resources is critical to ameliorating the impact of stroke on the limited resources of developing countries.

One way of ensuring acceptable levels of care is through the use of standard, evidence-based, clinical practice guidelines (CPGs). Several international CPGs for the management of acute ischaemic stroke have been published within the past decade, primarily by professional service organisations in developed countries [13-23]. However, the degree to which physicians in Barbados (and indeed, in the entire English-speaking Caribbean) adhere to these evidence-based recommendations is unknown. If implemented and managed appropriately, national disease surveillance systems, like the BNR, are an ideal tool for monitoring quality of acute and/or follow-up care. There are very few ongoing stroke surveillance systems in the Caribbean region with published data, particularly for small-island nation states, and none that we could find providing data on treatment. Here we report on the monitoring of acute hospital care for ischaemic stroke in 2010-2013 in Barbados. This work provides the impetus for the attainment of higher standards of stroke care and, by the provision of these baseline data, will allow monitoring of future public health interventions. Also, as the only Caribbean country continuously monitoring national stroke incidence, conducting the first such study of stroke management, we provide important information for other countries in the region.

\section{Methods \\ Aim, design and setting}

This study aimed to compare documented stroke management practices recorded by the national stroke registry with time-appropriate international stroke management guidelines, in order to identify areas for improvement, where relevant.

\section{Evidence for guidelines and current practice}

Online databases (UpToDate, Medline, Pubmed and Trip) and four key websites for Caribbean, UK, American and global healthcare guidance were systematically searched for evidence-based recommendations for the management of acute ischaemic stroke (see Appendix), with the final search made on 18 December 2015. Only CPGs published prior to or including the final year of the study (2013) were included, and recommendations from the most current versions applicable during the study period (2010-2013) were identified. This information was summarised into a general consensus from the various CPGs.

Key informant interviews using an informal, semi-structured approach were conducted with acute care physicians to establish current clinical practice for acute stroke in Barbados.

\section{Data source}

Data on stroke management practice were obtained from patients registered with the BNR-Stroke who had been treated at the single tertiary public hospital in Barbados, the Queen Elizabeth Hospital (QEH). The following anonymised data items on acute stroke events treated at the QEH and the management of patients in the acute setting for 2010-2013 (four of the first five full years of registry data collection ${ }^{1}$ ), were extracted from the BNR-Stroke database: patient demographic information, past medical history, drug history and medical management including admission status, clinical assessments, primary diagnostic tests and vascular imaging (all strokes), specialist evaluations and tests, and pharmacological management on arrival at hospital and on discharge (ischaemic strokes only). In the absence of a stroke unit, hospital ward information was collected to illustrate what proportion of patients received intensive care vs medical ward care. 


\section{Statistical analyses}

The number of ischaemic strokes managed at the QEH between 2010 and 2013, mean age, sex and the proportion of patients having the following specific clinical assessments and tests performed were assessed: Glasgow Coma Scale (GCS) score, neurological testing, swallow tests, ECGs and neuroimaging. In addition, the number and proportion of patients having specialist consultations, as well as those being prescribed recommended medications (acutely and on discharge), were assessed. Results were compared with the evidence-based recommendations available up to 2013. In this descriptive study we chose to minimise the number of formal statistical tests performed; where relevant, a test for difference in proportions was used. Data analyses were completed using Stata version 12 (StataCorp., College Station, TX, USA).

\section{Results}

Key informant interviews revealed the absence of a stroke unit and a local stroke management protocol, or one specific international CPG to which all physicians adhered. There was no one standard used by all hospital staff.

\section{Data description and patient outcomes}

There were 2431 acute stroke events registered with the BNR-Stroke between 1 January 2010 and 31 December 2013. Most were managed at the QEH (2001; 82.3\%), while the remainder either died before reaching the healthcare setting $(408 ; 16.8 \%)$ or were managed in the community (22;0.9\%). Of the 2001 hospitalised patients, 1735 had data fully abstracted by the BNR team (86.7\%), while the remaining $13.3 \%$ were excluded from analyses as they lacked complete information.

The patients' ages ranged from 18 to 104 years (mean 69.7 years; SD 15.1), with slightly more females affected than males (51.7\% vs $48.3 \%)$. Ward information was documented for 1722 patients (99.3\%), most of whom (1649; 95.8\%) were managed initially by emergency physicians. Over $75 \%$ of these were admitted to general medical wards for continued management, with 109 (6.6\%) admitted to intensive care units. The remainder were managed on other wards, died in the emergency department $(1.2 \%)$ or were discharged home (17.8\%). Ischaemic stroke was the predominant sub-type (1406; 81.0\%), 268 (15.4\%) were haemorrhagic strokes and $61(3.5 \%)$ unclassified. Almost three-quarters were discharged from the hospital alive (1007; 71.6\%).

\section{Systematic review of stroke care guidelines}

The six CPGs for the management of patients with acute stroke that were publicly available during 2010-2013 included those for the UK [13-16], the USA (with separate guidelines for the management of ischaemic [17] and haemorrhagic [18] stroke in adults), Canada [19, 20], the European Union [21, 22] and Australia [23]. The CPGs all made similar recommendations for the management of acute ischaemic stroke [13-23] summarised under the following headings: (i) Priority response and acute phase care by emergency medical services (EMS) and direct transport to nearest stroke care facility; (ii) clinical diagnosis and management in acute stroke unit; (iii) primary diagnostic tests and vascular imaging; (iv) specialist evaluations; (v) pharmacological management; (vi) surgical interventions; (vii) rehabilitation. In 2010-2013, the QEH stroke unit was not yet implemented and the BNR did not collect information on EMS care. Therefore only parameters (iii) $-(\mathrm{v})$ could be adequately assessed using BNR-Stroke data (see below). The recommendations from each of these three parameters are summarised in Table 1. (A planned future evaluation of the recently implemented stroke unit will assess all seven parameters.)

\section{Primary diagnostic tests and vascular imaging (all strokes; $N=1735$ )}

Following clinical diagnosis of stroke, urgent neuroimaging was recommended by all CPGs [13-23] to identify stroke type and guide treatment. Computed tomography $(\mathrm{CT})$ was the primary diagnostic scan recommended (lower cost and shorter duration), but most guidelines noted that magnetic resonance imaging (MRI), if available, was useful for detecting early signs of ischaemia. Additional vascular imaging (carotid ultrasound examination, cerebral/carotid angiography) were also recommended [13-23].

Table 1 Summary of guideline recommendations - diagnosis, specialist assessments and pharmacological management

\begin{tabular}{|c|c|}
\hline Recommendations & Further information \\
\hline \multicolumn{2}{|c|}{ Direct admission to specialised stroke unit } \\
\hline Diagnostic tests & Neuroimaging (non-contrast CT/MRI) \\
\hline Secondary diagnostic tests & $\begin{array}{l}\text { ECG and other cardiac investigations } \\
\text { if indicated } \\
\text { Vascular imaging, e.g. CT/MR angiography } \\
\text { and duplex ultrasonography }\end{array}$ \\
\hline Specialists' assessments & $\begin{array}{l}\text { Neurologist, nutritionist, physiotherapist, } \\
\text { speech therapist, rehabilitation therapists }\end{array}$ \\
\hline \multicolumn{2}{|c|}{ Pharmacological management } \\
\hline Thrombolysis & $\begin{array}{l}\text { Intravenous alteplase within 3-4.5 h of } \\
\text { symptoms for fibrinolysis }\end{array}$ \\
\hline Antiplatelet therapy & $\begin{array}{l}\text { Aspirin (acetyl salicylic acid) primarily } \\
\text { (alternates: Clopidogrel or Dipyridamole) } \\
\text { Acute use (associated with slight reduction } \\
\text { in mortality and morbidity) } \\
\text { Prescribed on discharge (secondary } \\
\text { prophylaxis) }\end{array}$ \\
\hline Anticoagulants & $\begin{array}{l}\text { Avoid routine use except if at risk of } \\
\text { thromboembolism }\end{array}$ \\
\hline Statins & $\begin{array}{l}\text { Avoid routine use } \\
\text { Advised for secondary prophylaxis }\end{array}$ \\
\hline
\end{tabular}


The only form of neuroimaging available at the QEH was the CT brain scan, with unenhanced CT scans performed for most patients (1646; 94.9\%); 1117 of them (67.9\%) within $24 \mathrm{~h}$ of hospital arrival. Any MRIs were done privately (and non-acutely); these were performed for only $18(1.0 \%)$ patients. Vascular imaging was also performed infrequently (Table 2).

\section{Specialist evaluations (ischaemic strokes; $N=1406$ )}

All six CPGs [13-23] advocated multidisciplinary management, requiring patients to be evaluated within the first few hours of admission by a number of specialists (for example neurologist, dietician, physiotherapist) to identify their specific needs for acute care, nutrition and rehabilitation.

For the 1406 ischaemic stroke patients, neurological examinations, including dysphagia screening (776; 55.2\%) were performed primarily by physicians who were not neurologists. Less than half of the patients had documentation of neurologist consultations (117; 44.5\%), 50.2\% (409) were seen by physiotherapists and very few received other specialist assessments (Table 3).

\section{Pharmacological management (ischaemic strokes)}

This study focused on acute treatment (given within $24 \mathrm{~h}$ of the onset of stroke symptoms or within $24 \mathrm{~h}$ of arrival at QEH) and drugs prescribed on discharge.

\section{Thrombolysis}

All CPGs [13-23] recommended that eligible patients receive the intravenous thrombolytic agent alteplase within $3-4.5 \mathrm{~h}$ of stroke symptom onset. Only six patients received intravenous thrombolysis between 2010 and 2013 (Table 4), out of 903 potentially eligible patients (Fig. 1).

\section{Antiplatelet therapy}

All CPGs recommended aspirin [13-23] or clopidogrel [16] as acute antithrombotic therapy to improve stroke

Table 2 Primary and secondary diagnostic tests at the Queen Elizabeth Hospital, Barbados, 2010-2013 ( $N=1735$ )

\begin{tabular}{|c|c|c|c|}
\hline \multicolumn{2}{|c|}{ Recommended investigation } & \multicolumn{2}{|c|}{ Tests performed } \\
\hline & & \multirow{2}{*}{$\begin{array}{l}\text { Number } \\
1646\end{array}$} & \multirow{2}{*}{$\frac{\%}{94.9}$} \\
\hline $\mathrm{CT}$ scan & & & \\
\hline MRI & & 18 & 1.0 \\
\hline \multirow[t]{2}{*}{$C T$ scan/timing } & $\leq 24 \mathrm{~h}$ & 1117 & 67.9 \\
\hline & $>24 \mathrm{~h}$ & 496 & 30.2 \\
\hline \multicolumn{2}{|c|}{ Electrocardiography } & 1527 & 88.0 \\
\hline \multicolumn{2}{|c|}{ Echocardiography } & 55 & 3.2 \\
\hline \multicolumn{2}{|c|}{ Cerebrovascular angiography } & 36 & 2.1 \\
\hline \multicolumn{2}{|c|}{ Carotid ultrasound } & 32 & 1.8 \\
\hline \multicolumn{2}{|c|}{ Carotid artery angiography } & 14 & 0.8 \\
\hline
\end{tabular}

Table 3 Specialist consultations for ischaemic stroke patients at the Queen Elizabeth Hospital, Barbados, 2010-2013

\begin{tabular}{llll}
\hline Specialist consultation $^{\mathrm{a}}$ & $\mathrm{N}^{\mathrm{b}}$ & \multicolumn{2}{l}{ Patients assessed } \\
\cline { 3 - 4 } & & No. & $\%$ \\
\hline Physiotherapist & 815 & 409 & 50.2 \\
Neurologist evaluation & 263 & 117 & 44.5 \\
Cardiologist & 153 & 30 & 19.6 \\
Speech and language pathologist & 263 & 29 & 11.0 \\
Occupational therapist & 170 & 17 & 10.0 \\
Rehabilitation specialist & 139 & 13 & 9.4 \\
Neurosurgeon evaluation & 0 & 0 & 0 \\
\hline
\end{tabular}

${ }^{\mathrm{a}}$ Data on nutritionist consultations were not abstracted by the registry

${ }^{\mathrm{b}}$ Total number of patients for whom data were available

outcome. Continuation of the drug or combinations of the drug with dipyridamole [14] after discharge was advised for secondary prophylaxis against recurrence. Alternative agents recommended for those intolerant of aspirin, or for whom it was contraindicated, included clopidogrel and dipyridamole [21, 22]. In Barbados, less than $50 \%$ of hospitalised ischaemic stroke patients were documented as having received aspirin acutely or upon discharge. Other antiplatelet drugs available at the QEH during 2010-2013 were clopidogrel, dipyridamole, and Aggrenox (aspirin plus modified dipyridamole); however, these were infrequently prescribed $(<5 \%$; Table 4$)$.

Of the $903(64.2 \%)$ ischaemic stroke patients who received a CT scan within 24 h, only $40.0 \%$ were documented as having been given aspirin within that time (Fig. 1). The case fatality in ischaemic stroke patients who had been documented as having received aspirin acutely (24.7\%; 95\% CI 20.3-29.1) was statistically significantly lower than those who had not $(32.8 \%$; $95 \% \mathrm{CI}$ 28.9-36.8; $p=0.009$ ) (Fig. 1).

Table 4 Drugs prescribed for hospitalised ischaemic stroke patients, Barbados, 2010-2013

\begin{tabular}{|c|c|c|c|c|c|}
\hline \multirow[t]{2}{*}{ Drug category } & \multirow[t]{2}{*}{ Drug name } & \multicolumn{2}{|c|}{$\begin{array}{l}\text { Prescribed } \\
\text { acutely }\end{array}$} & \multicolumn{2}{|c|}{$\begin{array}{l}\text { Prescribed on } \\
\text { discharge }\end{array}$} \\
\hline & & No. & $\%$ & No. & $\%$ \\
\hline \multirow[t]{4}{*}{ Antiplatelet drugs } & Aspirin & 521 & 37.0 & 670 & 47.7 \\
\hline & Clopidogrel & 50 & 3.6 & 66 & 4.7 \\
\hline & Dipyridamole & $<10$ & $<1$ & 13 & 0.9 \\
\hline & Aggrenox & $<10$ & $<1$ & 12 & 0.9 \\
\hline Fibrinolytic & Alteplase & $<10$ & $<1$ & $\mathrm{n} / \mathrm{a}$ & \\
\hline \multirow[t]{3}{*}{ Anticoagulants } & Warfarin & 16 & 1.1 & 49 & 3.5 \\
\hline & Heparin (subcutaneous) & 333 & 23.7 & $\mathrm{n} / \mathrm{a}$ & \\
\hline & $\begin{array}{l}\text { Heparin (low molecular } \\
\text { weight) }\end{array}$ & 44 & 3.1 & 32 & 2.3 \\
\hline Statins & Simvastatin, Atorvastatin & 304 & 21.6 & 681 & 48.4 \\
\hline
\end{tabular}




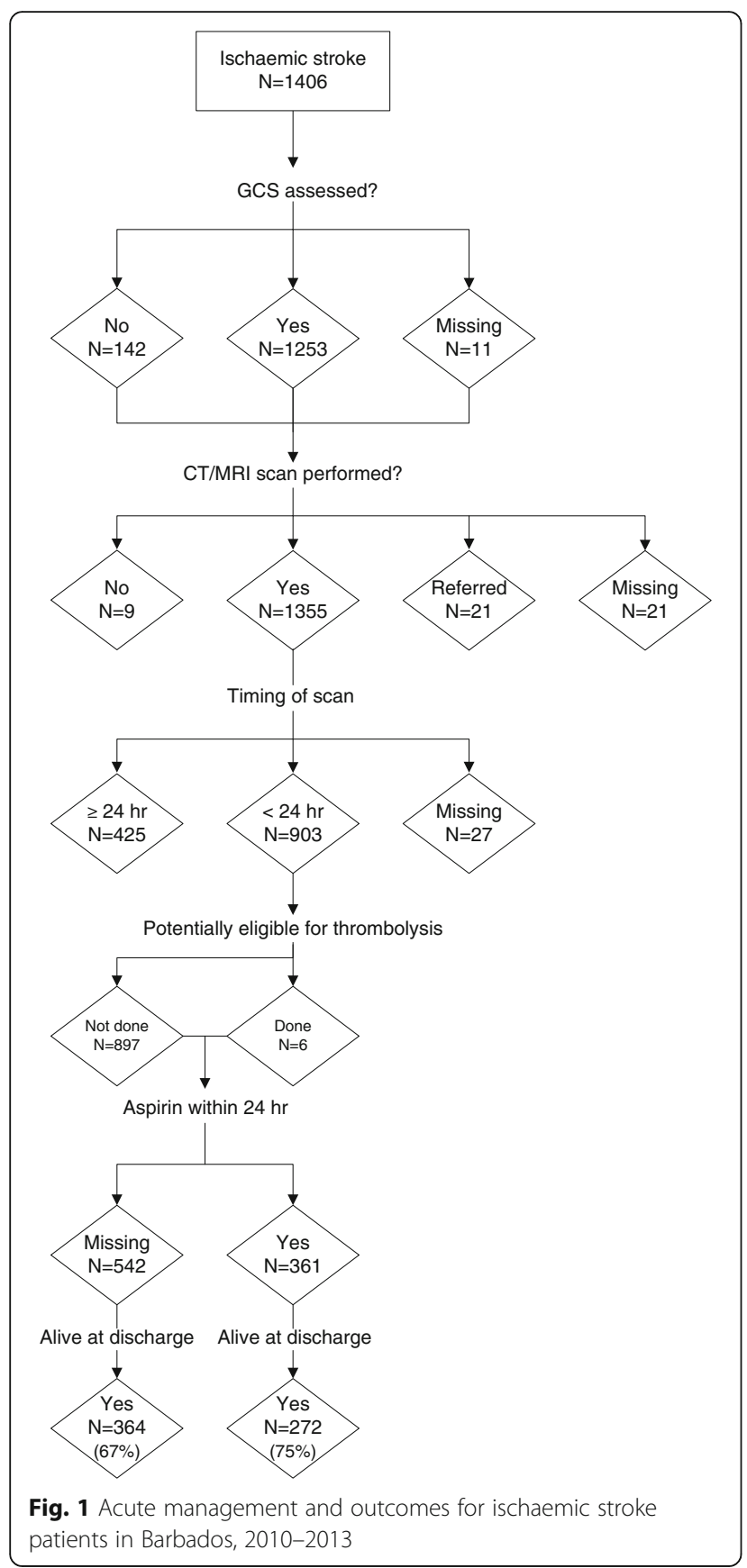

\section{Anticoagulants}

All guidelines [13-23] discouraged the use of anticoagulants except in selected patients which included those at risk of thromboembolic phenomena (e.g. with atrial fibrillation or thrombophilias) [13-23] and patients with cerebral venous sinus thrombosis [14]. In Barbados, $1.1 \%$ of ischaemic stroke patients were given warfarin acutely, $3.5 \%$ received the drug on discharge (Table 4 ) and 48 patients were chronic users (data not shown). In each successive year, acute use was reduced; patients continuing warfarin likely had high risk of recurrent stroke (e.g. co-morbid atrial fibrillation: 107 patients). Acute use of heparin was documented for 377 (26.8\%) patients (Table 4).

\section{Statins}

Most guidelines did not recommend the initiation of statins in the acute setting, but supported their continuation for patients with prior chronic use. All guidelines [13-23] recommended the subsequent use of statins as secondary prophylaxis against recurrent ischaemic stroke. Acutely, 304 (21.6\%) hospitalised patients in Barbados were documented as having received statins while 681 (48.4\%) were prescribed a statin on discharge (Table 4 ).

\section{Discussion}

Our study showed that, during 2010-2013, the diagnostic approach to acute stroke at the QEH in Barbados was consistent with international CPGs. Stroke was diagnosed clinically, and almost all stroke patients (95\%) had documented CT brain imaging during their hospital stay; $68 \%$ within $24 \mathrm{~h}$. Unfortunately, the exact timing of CT scans was not collected by the registry (which just has a checkbox for "CT within 24 hrs"), thus we were unable to determine the proportion of patients scanned within 3-4.5 h of symptom onset. Our study showed that, in contrast to what was found for diagnostics, the management of patients with ischaemic stroke was not in keeping with CPG recommendations, as these patients were managed mainly on general medical wards during the study period. During the study period, few persons were documented as having received the specialist consultations recommended by the CPGs as part of routine ischaemic stroke care. Further, thrombolysis was seldom performed, with fewer than 10 patients thrombolysed in 2010-2013. In Barbados, few patient records (37\%) documented the acute use of aspirin, despite evidence in support of aspirin therapy causing minor improvements in dependence and mortality when given within $24 \mathrm{~h}$ of the event. Fewer records than expected $(<50 \%)$ showed that aspirin had been given as secondary prophylaxis on discharge. Likewise, there was limited documentation of prescription of statins for secondary prophylaxis of ischaemic stroke. Despite this, our study found that the overall proportion of hospitalised ischaemic stroke patients who survived to 30 days was $74.9 \%$, for a mean 30 -day case fatality of $24.9 \%$ (22.7-27.3).

Our rate of administration of CT scans within $24 \mathrm{~h}$ is similar to the $69 \%$ seen in Canada [24] and is commendable given the absence of a specific, written hospital stroke protocol. However, there is still considerable room for improvement, especially when compared with other countries such as Australia, where $89 \%$ of patients receive CT scans within $24 \mathrm{~h}$ [25]. 
In Barbados, late patient presentation to the hospital, the lack of a protocol for administering alteplase for stroke, and the absence of a stroke unit in which to subsequently manage patients, may have collectively accounted for the low rates of thrombolysis, which have also been noted by other low-resourced countries [26, 27]. Even hospitals equipped with better developed stroke care systems experience challenges, however. The 2011 Australian National stroke audit found only $36 \%$ of patients presented to facilities within $3 \mathrm{~h}$ of stroke symptom onset, only $7 \%$ of patients were thrombolysed and less than $60 \%$ of patients were managed in stroke units [28]. Similarly, in Canada, only $25 \%$ of hospitals providing acute stroke care had a specialised programme; $17 \%$ had stroke units and $50 \%$ of patients presented to the hospital outside the thrombolysis eligibility period [24]. Conversely, other countries (e.g. England, Wales and Northern Ireland) found marked improvements in CT scan times (43\% in $1 \mathrm{~h}, 80 \%$ in $12 \mathrm{~h}$ ) and thrombolysis rates (75\%) [29]. Award programmes such as the AHA/ASA 2013 'Get with the guidelines - Stroke ${ }^{\text {(R) }}$, have proved useful in driving improvement in some American hospitals. Almost $60 \%$ of hospitals participating in this initiative achieved the door-to-needle target for thrombolysis of $60 \mathrm{~min}$ or less [30].

Interviews with the sole neurologist at the hospital in Barbados suggested that our observations on low proportions of aspirin and other key medications are unlikely to be a true reflection of clinical practice, more likely being due to omissions in documentation. Improved documentation in medical records is clearly needed, not only for surveillance, medico-legal and research purposes, but also to establish a true reflection of patient management and ensure best practices are being consistently followed. Care by a specialised stroke team and, where possible, care in a dedicated stroke unit (both recommended as first-line treatment by all CPGs) are associated with reduced mortality and patient disability [29, 31, 32].

Low levels of specialist input and rehabilitation therapy likely reflect a lack of CPG guidance or poor documentation, as well as limited resources and/or ineffective resource allocation. For example, there was only one neurologist and a limited number of therapists working at the hospital, presenting an impractical patient load. Only 117 patients had documented evidence of referral to the neurologist and a mere 37 to a speech and language therapist (Table 3). Although improved patient outcomes are associated with adherence to CPGs [25, 33], the goal of such guidelines is not strict adherence but rather to provide physicians with minimum basic standards and proven treatment algorithms that can be tailored to the individual needs of specific patients. However, CPGs for developed countries may not always be appropriate or implementable in low-resourced, small-island developing states such as Barbados. For example, general physicians managing stroke patients may only refer difficult cases for specialist consultation and may not recognise subtle deficits that may be critical to determining patient management. The lack of expert assessment of dysphagia by neurologists could lead to high levels of aspiration pneumonia and higher mortality.

Data from a 2006 study comparing acute stroke outcomes for Black Barbadians and a similar population in South London revealed relatively poor first-ever stroke survival for the island (hazard ratio 1.99; 95\% CI 1.23-3.21, $p=0.005$ ) [34]. Comparisons of local acute stroke management and post-stroke care subsequent to discharge were beyond the scope of this study, which only analysed data collected by the national stroke registry. The case fatality we found, however, is comparable with the estimated worldwide 30-day case fatality after first ischaemic stroke (16-23\%) [33] and with rates for other Caribbean countries, such as Trinidad (29\%) [27], suggesting that ischaemic stroke patients in Barbados received at least a similar standard of care to patients in countries with similar resources. However, improvement is needed given the far lower case fatalities observed in developed countries during the same period (e.g. United Kingdom: $12.9 \%$ [29], Canada, excluding Québec: 15\% [29], USA: 4.2\% [26]).

The limitations of our study included the retrospective (secondary) use of prospectively collected data acquired solely from hospital records, which firstly limited our analyses to only those parameters already collected by the registry. Secondly, our analysis would have failed to capture any undocumented consultations and treatments. Poor documentation of key parameters, e.g. aspirin use, may have skewed the results obtained, and it is impossible to determine whether care was actually omitted, or given but not recorded. Neither option reflects good clinical practice. The BNR team will continue their efforts to address the issue of poor documentation during professional development seminars for medical professionals. Future years of BNR data will be able to assess the impact of the stroke unit on stroke outcomes in Barbados.

\section{Conclusion}

Despite good adherence to diagnostic CPGs, there was a lack of evidence of adherence to standard CPGs for acute medical management of ischaemic stroke between 2010 and 2013 in Barbados, which may be due in part to the limited resources experienced by small-island developing nations. Mitigating the mortality and degree of morbidity experienced by patients is dependent on rapid appropriate management from the time of symptom onset. It is therefore essential that patients be aware of the symptoms of stroke, as well as the importance of 
reporting immediately to a medical facility for evaluation and subsequent management. This speaks to the need to educate the general public through health promotion information on stroke, as well as the direct education of at-risk patients by primary care providers.

The recent implementation of a stroke unit at the hospital, as well as future hospital stroke management protocols, will be important steps toward improving the standard of care at the QEH. Monitoring of these initiatives will be provided through continued stroke surveillance. Results from this study likely reflect practices in other similar, low-resource settings, with implications particularly for other Caribbean nations.

\section{Endnote}

${ }^{1}$ Note: data from 2009 were excluded, as it was the first registry year.

\section{Appendix}

\section{Details of literature search}

The following databases were searched online for published literature:

UpToDate.

PUBMED/MEDLINE.

Trip.

For 'grey' literature (i.e. technical and/or government reports which may not be published in the scientific literature), the following websites were reviewed:

Centers for Disease Control and Prevention (CDC), USA. http://www.cdc.gov/

World Health Organization (WHO). http:// www.who.int/en/

Caribbean Health Research Council (CHRC). http:// carpha.org

National Institute of Health for Clinical Excellence

(NICE). http://www.nice.org.uk/

The following terms were used for each database/web-

site search:

acute stroke management

ischaemic haemorrhagic stroke management

stroke best practice

stroke guidelines

stroke management guidelines

stroke treatment

stroke treatment recommendations

Databases and websites were last searched on 18

December 2015.

\section{Abbreviations}

AHA: American Heart Association; ASA: American Stroke Association; BNR: Barbados National Registry for Chronic Non-communicable Disease; CDC: Centers for Disease Control and Prevention; CHRC: Caribbean Health Research Council; CPG: Clinical practice guideline; CSN: Canadian Stroke Network; CT: Computed tomography; CVD: Cardiovascular disease; ECG: Electrocardiogram; EMS: Emergency medical services; ESO: European Stroke Organisation; GCS: Glasgow coma scale; HSF: Heart and Stroke
Foundation; ISWG: Intercollegiate Stroke Working Group; MRI: Magnetic resonance imaging; NCD: Non-communicable disease; NICE: National Institute for Health and Care Excellence; QEH: Queen Elizabeth Hospital; SD: Standard deviation; SIGN: Scottish Intercollegiate Guideline Network: UK: United Kingdom; USA: United States of America; WHO: World Health Organization

\section{Acknowledgements}

We thank all members of the BNR-Stroke team, without whose hard work and dedication this analysis would not have been possible. We are grateful to all the stroke registry stakeholders and data sources for their support, in particular the Barbados Ministry of Health, which has funded the BNR since its inception in 2008. Most importantly, we thank all stroke patients and their families for their co-operation in being part of the national stroke registry.

\section{Funding}

No additional funding support was received for this particular study, but the BNR is funded by the Barbados Ministry of Health. The funding body had no role in the design of the study or data analysis; co-author KSG is the Senior Medical Officer for NCDs at the Barbados Ministry of Health.

\section{Availability of data and materials}

The datasets used and analysed during the current study are available from the corresponding author on reasonable request.

\section{Authors' contributions}

AJMH, IRH, DOCC, KSG and AMCR conceived and designed the study; LM and TPM supervised data collection and reviewed data for quality control; IRH, AMCR and TB prepared the dataset; ASHM and AMCR prepared the initial draft and analysed the initial dataset; LSC and DMC prepared subsequent drafts and performed later analyses; KSG, AJMH, DOCC, AMCR and IRH were major contributors in revising the manuscript. All authors read and approved the final manuscript.

Ethics approval and consent to participate

Individual consent to participate was not applicable as this study comprised a secondary analysis of prospectively collected, anonymised national stroke registry data from the BNR, which receives annual ethics approval from the Barbados Ministry of Health/University of the West Indies' Institutional Review Board and from the QEH Executive Board Ethics Sub-Committee.

Competing interests

The authors declare that they have no competing interests.

\section{Publisher's Note}

Springer Nature remains neutral with regard to jurisdictional claims in published maps and institutional affiliations.

\section{Author details}

'George Alleyne Chronic Disease Research Centre, Caribbean Institute for Health Research, The University of the West Indies, Cave Hill Campus, Barbados. ${ }^{2}$ Ministry of Health, Frank Walcott Building, Culloden Road, Bridgetown, Barbados

Received: 22 October 2017 Accepted: 11 May 2018

Published online: 22 May 2018

References

1. Truelsen T, Begg S, Mathers C. The global burden of cerebrovascular disease. 2002. http://www.who.int/healthinfo/statistics/bod_ cerebrovasculardiseasestroke.pdf. Accessed 8 Jan 2014.

2. WHO. Cardiovascular diseases (CVDs). http://www.who.int/mediacentre/ factsheets/fs317/en/. Accessed 6 Jun 2017.

3. Mathers $C D$, Lopez AD, Murray CJL. The burden of disease and mortality by condition: data, methods, and results for 2001. In: The global burden of disease and risk factors. Chapter 3. 2003. https://www.ncbi.nlm.nih.gov/ books/NBK11808/. Accessed 8 Jan 2014.

4. WHO. Action plan for the global strategy for the prevention and control of noncommunicable diseases. World Health Organization. 2008-2013. http.// www.who.int/nmh/publications/9789241597418/en/. Accessed 8 Jan 2014. 
5. Barbados Statistical Service. 2010 Population and housing census report, vol. 1. Barbados: Government Printing Department. http://www.barstats.gov.bb/ files/documents/PHC_2010_Census_Volume_1.pdf. Accessed 15 Sep 2013.

6. WHO. Noncommunicable diseases country profiles 2011. World Health Organization. 2012. http://www.who.int/nmh/publications/ncd_profiles2011/en/. Accessed 8 Jan 2014

7. World Population review. Barbados Population (2017-11-10). http:// worldpopulationreview.com/countries/barbados-population/ Accessed 15 Dec 2017.

8. Rose AM, Hambleton IR, Jeyaseelan SM, Howitt C, Harewood R, Campbell J, et al. Establishing national non-communicable disease surveillance in a developing country: a model for small island nations. Pan Am J Public Health. 2016;39:76-85.

9. $\mathrm{AMC}$ R. Understanding the epidemiology of cardiovascular disease in a small-island nation with an ageing population. Cave Hill: PhD thesis, The University of the West Indies; 2017.

10. Bath PM, Lees KR. ABC of arterial and venous disease. Acute stroke. BMJ. 2000;320:920-3.

11. Tarride J-E, Lim M, DesMeules M, Luo W, Burke N, O'Reilly D, et al. A review of the cost of cardiovascular disease. Can J Cardiol. 2009;25:e195-202.

12. Taylor TN, Davis PH, Torner JC, Holmes J, Meyer JW, Jacobson MF. Lifetime cost of stroke in the United States. Stroke. 1996;27:1459-66.

13. Party ISW. National clinical guideline for stroke. 4th ed. London: Royal College of Physicians; 2012. https://www.strokeaudit.org/Guideline/ Historical-Guideline/National-Clinical-Guidelines-for-Stroke-fourth-edi.aspx. Accessed 8 Jan 2014

14. National Institute for Health and Care Excellence. Stroke and transient ischaemic attack in over 16s: diagnosis and initial management. 2008. http://nice.org.uk/guidance/cg68. Accessed 18 Dec 2015.

15. National Institute for Health and Care Excellence. Alteplase for treating acute ischaemic stroke. 2012. http://www.nice.org.uk/guidance/ta264. Accessed 04 Dec 2015.

16. Management of patients with stroke or TIA: assessment, investigation, immediate management and secondary prevention (SIGN Guideline No 108). http://www.sign.ac.uk/sign-108-management-of-patients-with-strokeor-tia-assessment-investigation-immediate-management-a.html. Accessed 8 Jan 2014.

17. Adams HP Jr, del Zoppo G, Alberts MJ, Bhatt DL, Brass L, Furlan A, et al. Guidelines for the early management of adults with ischemic stroke: a guideline from the American Heart Association/American Stroke Association stroke council, clinical cardiology council, cardiovascular radiology and intervention council, and the atherosclerotic peripheral vascular disease and quality of care outcomes in research interdisciplinary working group. Circulation. 2007;115:e478-534

18. Broderick J, Connolly S, Feldmann E, Hanley D, Kase C, Krieger D, et al. Guidelines for the management of spontaneous intracerebral hemorrhage in adults: 2007 update: a guideline from the American Heart Association/ American Stroke Association stroke council, high blood pressure research council, and the quality of care and outcomes in research interdisciplinary working group. Circulation. 2007;116:e391-413.

19. Canadian Best Practice Recommendations for Stroke Care (updated 2008). https://www.ncbi.nlm.nih.gov/pmc/articles/PMC2586332/pdf/20081202. 1s00001pS1.pdf. Accessed 31 Jan 2014.

20. Canadian Best Practice Recommendations for Stroke Care (updated 2013). http://canadianstrokenetwork.ca/en/wp-content/uploads/2014/08/CSBPTaking-Action-Resource-EN.pdf. Accessed 15 Dec 2015.

21. European Stroke Organisation (ESO) Executive Committee and the ESO Writing Committee. 2008. Guidelines for management of ischaemic stroke and transient ischaemic attack 2008. http://www.karger.com/Article/Pdf/ 131083. Accessed 14 Feb 2014.

22. European Stroke Organisation. Karolinska stroke update 2008. Final draft per 2008. ESO GC statement on revised guidelines for intravenous thrombolysis. http://www.congrex-switzerland.com/fileadmin/files/2013/eso-stroke/pdf/ ESO_Guideline_Update_Jan_2009.pdf. Accessed 8 Jan 2014.

23. National Institute of Clinical Studies. Emergency department stroke and transient ischaemic attack care bundle: information and implementation guide. Melbourne: National Health and Medical Research Council; 2009. http://www.nhmrc.gov.au/_files_nhmrc/publications/attachments/cp116_ app_g_rosier_scale.pdf. Accessed 9 Jan 2014.

24. Canadian Institute for Health Information (C IHI). Health indicators 2013: definitions, data sources and rationale. Ottawa (ON): Canadian Institute for
Health Information (CIHI). May 2013. http://publications.gc.ca/collections/ collection_2013/icis-cihi/H115-67-2013-eng.pdf. Accessed 15 Jun 2017.

25. Hubbard IJ, Harris D, Kilkenny MF, Faux SG, Pollack MR, Cadilhac DA. 2012. Adherence to clinical guidelines improves patient outcomes in Australian audit of stroke rehabilitation practice. Arch Phys Med Rehabil. 2012;93:965-71.

26. OECD. In-hospital mortality following stroke. In: Health at a glance 2011: OECD Indicators, OECD Publishing; 2011. https://www.oecd.org/els/healthsystems/49105858.pdf. Accessed 14 Feb 2014.

27. Mahabir D, Bickram L, Gulliford MC. Stroke in Trinidad and Tobago: burden of illness and risk factors. Rev Panam Salud Publica. 1998:4:233-7.

28. National Stroke Foundation. National stroke audit - acute services clinical audit report 2011. Melbourne, Australia. https://informme.org.au/stroke-data/ acute-audits. Accessed 8 Jan 2014.

29. Rudd AG. Hoffman a, Irwin P, Pearson M, Lowe D, intercollegiate working Party for Stroke. Stroke units: research and reality. Results from the national sentinel audit of stroke. Qual Saf Health Care. 2005;14:7-12.

30. American Heart Association. 2015. Get with the guidelines (r)-stroke. http:// www.heart.org/HEARTORG/HealthcareResearch/GetWithTheGuidelines/ GetWithTheGuidelines-Stroke/Get-With-The-Guidelines-Stroke-Overview_ UCM_308021_Article.jsp\#.VnQ7Qr8YNYM. Accessed 18 Dec 2015.

31. Stroke Unit Trialists' Collaboration. Organised inpatient (stroke unit) care for stroke. Cochrane Database Syst Rev. 2013;11:CD000197.

32. Heart and Stroke Foundation. 2014 Stroke report: together against a rising tide: advancing stroke systems of care. https://www.heartandstroke.ca/-/ media/pdf-files/canada/stroke-report/hsf-stroke-report-2014.ashx?la= en\&hash=4FD2B18A0EEDA2A193EFDBBDB983F23B1FBD570D. Accessed 15 Dec 2015.

33. Oh SW, Lee HJ, Chin HJ, Hwang Jl. 2011. Adherence to clinical practice guidelines and outcomes in diabetic patients. Int J Qual Health Care. 2011; 23:413-9.

34. Wolfe CDA, Corbin DOC, Smeeton NC, Gay GHE, Rudd AG, Hennis AJ, et al. Estimation of the risk of stroke in black populations in Barbados and South London. Stroke. 2006:37:1986-90.

\section{Ready to submit your research? Choose BMC and benefit from:}

- fast, convenient online submission

- thorough peer review by experienced researchers in your field

- rapid publication on acceptance

- support for research data, including large and complex data types

- gold Open Access which fosters wider collaboration and increased citations

- maximum visibility for your research: over $100 \mathrm{M}$ website views per year

At BMC, research is always in progress.

Learn more biomedcentral.com/submissions 